In Crescendo. Institucional. 2016; 7(1): 90-100

Fecha de recepción: 6 de mayo de 2016

Fecha de aceptación: 17 de mayo de 2016

\title{
Aplicación del programa "Buena Ortografía sin Esfuerzo" PARA MEJORAR EL NIVEL ORTOGRÁFICO EN ALUMNOS DEL I CICLO DE la Escuela de Enfermería de la Universidad Católica Los Ángeles de Chimbote, 2010
}

\section{Application of the program "Buena Ortografía sin Esfuerzo" \\ TO IMPROVE THE SPELL LEVEL ON I CYCLE STUDENTS OF NURSING school at Universidad Católica los Ángeles de Chimbote, 2010}

Natalia Mávila Guzmán Rodríguez*

María Ysabel Moya Chávez**

\section{RESUMEN}

$\mathrm{E}^{1}$ presente estudio es de tipo experimental aplicativo. Tuvo como propósito diseñar un programa denominado "Buena Ortografía sin Esfuerzo" y se evaluó si la aplicación de este programa mejora el nivel ortográfico en alumnos del I ciclo de la Escuela de Enfermería de la Universidad Católica Los Ángeles de Chimbote. La población estuvo constituida por 37 estudiantes (19 de grupo experimental y 18 de grupo control) cuyas edades estuvieron comprendidas entre los 17 y 20 años. Se utilizó el diseño con grupo control pretest y postest. En el análisis comparativo se encontró que en el postest se comparó el promedio obtenido por el grupo experimental con el promedio obtenido por el grupo control, existiendo diferencia significativa entre dichos promedios $(\mathrm{p}<0.05)$

Palabras clave: nivel ortográfico, programa, universidad.

* Maestra en Educación con mención en problemas de aprendizaje de la Universidad Privada Antenor Orrego. Docente de la Escuela de Psicología de la Universidad Católica Los Ángeles de Chimbote.

** Doctora en Psicología. Docente en la sección de posgrado en Educación de la Universidad Privada Antenor Orrego. 


\section{ABSTRACT}

This study is an experimental application. The purpose of this study was to develop a program called "Good spelling no effort" to evaluate if the implementation of this program improves the spell level on I cycle students of nursing school at Universidad Católica Los Ángeles de Chimbote. The sample consisted of 37 students (19 experimental and 18 control group) which ages ranges into 17 and 20 years. It was used an experimental design with a control group pre test and post test. In the comparative analysis it was found that the average obtained by the experimental group with and the control group was compared in the post test had a significant difference between those averages $(\mathrm{p}<$ $0.05)$

Keywords: Spell level, program, university.

\section{INTRODUCCIÓN}

La realidad universitaria presenta a jóvenes en los inicios de su formación profesional con una serie de dificultades en el desarrollo de actitudes, adquisición de conocimientos y manejo de habilidades esperadas en el momento de culminar la educación básica de nivel secundario. Esta es una apreciación recurrente en las aulas universitarias, pues a diario se observan las deficiencias como resultado de una débil instrucción secundaria de aquellos alumnos que se encuentran en los primeros ciclos de las distintas carreras profesionales. Es el caso de aquellos alumnos que presentan dificultades con respecto a la escritura correcta de las palabras, es decir, a la ortografía.

Portilla (2008) ha podido observar durante sus años de experiencia docente que los ingresantes, sean de la especialidad que fuere, manifiestan una falta de competencia ortográfica realmente alarmante: no saben escribir correctamente ni siquiera las fechas. Y si evalúan a través de una prueba sencilla su manejo ortográfico, se podrá comprobar que carecen de las nociones mínimas de puntuación y tildación.

Así también, Cassany (citado en Morales y Fernández, 2004) refiere que en el contexto educativo formal, los docentes revelan, tanto formal como informalmente, cada vez que tienen la oportunidad, su preocupación por la adquisición por parte de los estudiantes de los conocimientos ortográficos. Esto se evidencia en las producciones escritas de estudiantes de los distintos niveles educativos, que dejan perplejos a los lectores por sus características.

Como se ha venido refiriendo, en el nivel de educación superior, principalmente, durante los primeros ciclos de estudio, se observa un gran número de alumnos que presenta serias dificultades en el área de la escritura expresiva, principalmente, en la ortografía, lo cual es producto de la falta de aprehensión de las reglas y de una instrucción deficiente.

Estas dificultades, sin embargo, han de ser superadas a través de programas de intervención para la mejora de la ortografía. De esta manera, el uso de técnicas pedagógicas y psicológicas, permiten el avance en tales alumnos con dificultades en la ortografía. 


\section{MATERIAL Y MÉTODOS}

El presente trabajo de investigación es de tipo experimental, aplicativa, pues considera la influencia de un estímulo sobre una variable dependiente a fin de que esta sea modificada (Hernández, Fernández y Baptista, 2006).

El diseño que se utilizó fue de tipo cuasi-experimental con dos grupos intactos, pretest y postest, que tiene el siguiente esquema:

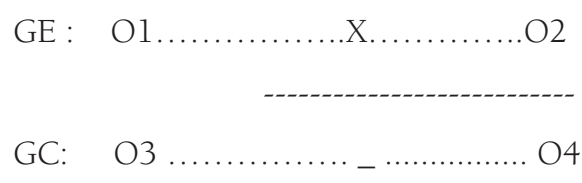

Donde:

GE: Grupo Experimental (representado por alumnos del I ciclo de la Escuela de Enfermería con Programa "Buena Ortografía sin Esfuerzo").

GC: Grupo Control (representado por alumnos del I ciclo de la Escuela de Enfermería sin Programa "Buena Ortografía sin Esfuerzo").

O1 O3: Pretest

O2 O4: Postest

X : Programa "Buena ortografía sin esfuerzo",

La población estuvo constituida por 37 alumnos matriculados del I ciclo de la Escuela de Enfermería que asistieron con regularidad a la Universidad Católica Los Ángeles de Chimbote en la sede de Trujillo. Las edades estuvieron comprendidas entre los 17 y 20 años. Para el presente estudio, la muestra estuvo formada por toda la población del estudio distribuida en 19 alumnos para el grupo experimental y 18 para el grupo control.

Para la recolección de datos se aplicó una prueba de ortografía (pretest-postest), elaborada por la autora de la presente investigación, a fin de determinar el nivel de ortografía de los alumnos del I ciclo de la Escuela de Enfermería. La prueba fue dividida en 4 partes: la primera se confeccionó con 7 oraciones incompletas, en las que se tenía que utilizar las letras "s", "c", "v", "b" para las respuestas correctas; en la segunda parte, se consideraron 7 palabras incompletas para las letras "b" y "v"; en la tercera parte, se plantearon 7 oraciones incompletas para las letras "c" y "s"; y, en la cuarta parte, 8 oraciones incompletas para ejercicios de tildación.

Por otro lado, se consideró para la calificación una puntuación de 0 a 29 puntos, en que el intervalo de 0 a 9 puntos asumido como un nivel malo; de 10 a 18 puntos, nivel regular, y 1 nivel bueno entre 19 y 29 puntos.

\section{Dimensiones}

1. Uso de las letras S, C, B, V.

2. Uso de las letras C y S.

3. Uso de las letras $\mathrm{V}$ y B.

4. Uso de tildes. 
Se aseguró la validez externa del instrumento por juicio de expertos presentando el instrumento a 4 expertos en el área a investigar, quienes con sus sugerencias brindaron mayor calidad y especificidad a los instrumentos.

Asimismo, para determinar la validez, en términos de consistencia interna, se utilizó el coeficiente producto momento de Pearson y para precisar con exactitud la correlación se usó el corrector de McNemar; quien sostiene que si el valor hallado es mayor a 0,21 el ítem se considera válido. Los resultados estuvieron entre 0,26 y 0,81 por lo que se determina que el instrumento presentó una buena validez.

El instrumento fue sometido a la prueba de confiabilidad alfa de Cronbach, y se obtuvo un valor satisfactorio de a $>0,92$; por consiguiente, el instrumento de evaluación está apto para su aplicación.

En cuanto al procedimiento realizado en la presente investigación, se diseñó el programa "Buena Ortografía sin Esfuerzo" tomando en cuenta los indicadores del nivel ortográfico de la presente investigación.

Se aplicó la prueba de ortografía a los alumnos de I ciclo, a fin de determinar el nivel ortográfico de entrada.

Se aplicó el programa "Buena Ortografía sin Esfuerzo" a alumnos del I ciclo de la Escuela de Enfermería de la Universidad Católica Los Ángeles de Chimbote, sede Trujillo, previa información y consentimiento de los alumnos.

Después de ejecutado el programa, se aplicó la prueba de ortografía a los alumnos de I ciclo a fin de determinar el nivel ortográfico de salida.

Para el análisis de los datos, se ejecutó la prueba estadística "t" de student para determinar la efectividad del programa.

\section{RESULTADOS}

Tabla 1.Niveles de ortografía alcanzado en el pretest y postest del grupo experimental y control de los alumnos del I ciclo de la Escuela de Enfermería de la Universidad Católica Los Ángeles de Chimbote, sede Trujillo, 2010.

\begin{tabular}{lcccccccc}
\hline \multirow{2}{*}{$\begin{array}{l}\text { NIVELES DE } \\
\text { ORTOGRAFÍA }\end{array}$} & \multicolumn{3}{c}{ GRUPO EXPERIMENTAL } & \multicolumn{3}{c}{ GRUPO CONTROL } \\
\cline { 2 - 8 } & \multicolumn{2}{c}{ PRETEST } & \multicolumn{2}{c}{ POSTEST } & \multicolumn{2}{c}{ PRETEST } & \multicolumn{2}{c}{ POSTEST } \\
\hline MALA & 13 & 68.42 & 0 & 0 & 03 & 16.67 & 04 & 22.22 \\
REGULAR & 06 & 31.58 & 03 & 15.79 & 15 & 83.33 & 14 & 77.78 \\
BUENA & 0 & 0 & 16 & 84.21 & 0 & 0 & 0 & 0 \\
TOTAL & 19 & 100.0 & 19 & 100.0 & 18 & 100.0 & 18 & 100.00
\end{tabular}

Fuente: Prueba de ortografía, elaborada por Guzmán N. Trujillo, 2010. 
Tabla 2. Niveles de ortografía alcanzado en el pretest y postest del grupo experimental y control en el uso de la letra "v" de los alumnos del I ciclo de la Escuela de Enfermería de la Universidad Católica Los Ángeles de Chimbote, sede Trujillo, 2010.

\begin{tabular}{|c|c|c|c|c|c|c|c|c|}
\hline \multirow{3}{*}{$\begin{array}{l}\text { USO DE LA } \\
\text { LETRA "V" }\end{array}$} & \multicolumn{4}{|c|}{ GRUPO EXPERIMENTAL } & \multicolumn{4}{|c|}{ GRUPO CONTROL } \\
\hline & \multicolumn{2}{|c|}{ PRETEST } & \multicolumn{2}{|c|}{ POSTEST } & \multicolumn{2}{|c|}{ PRETEST } & \multicolumn{2}{|c|}{ POSTEST } \\
\hline & N. ${ }^{o}$ & $\%$ & $\mathrm{~N}^{\circ}$ & $\%$ & N. ${ }^{\circ}$ & $\%$ & $\mathrm{~N}^{\circ}$ & $\%$ \\
\hline MALA & 12 & 63.16 & 0 & 0 & 01 & 5.56 & 0 & 0 \\
\hline REGULAR & 07 & 36.84 & 04 & 21.05 & 17 & 94.44 & 18 & 100.0 \\
\hline BUENA & 0 & 0 & 15 & 78.95 & 0 & 0 & 0 & 0 \\
\hline TOTAL & 19 & 100.0 & 19 & 100.0 & 18 & 100.0 & 18 & 100.0 \\
\hline
\end{tabular}

Fuente: Prueba de ortografía, elaborada por Guzmán N. Trujillo, 2010.

Tabla 3. Niveles de ortografía alcanzado en el pretest y postest del grupo experimental y control en el uso de la letra "b" de los alumnos del I ciclo de la Escuela de Enfermería de la Universidad Católica Los Ángeles de Chimbote, sede Trujillo, 2010.

\begin{tabular}{|c|c|c|c|c|c|c|c|c|}
\hline \multirow{3}{*}{$\begin{array}{c}\text { USO DE LA LETRA } \\
\text { "B" }\end{array}$} & \multicolumn{4}{|c|}{ GRUPO EXPERIMENTAL } & \multicolumn{4}{|c|}{ GRUPO CONTROL } \\
\hline & \multicolumn{2}{|c|}{ PRETEST } & \multicolumn{2}{|c|}{ POSTEST } & \multicolumn{2}{|c|}{ PRETEST } & \multicolumn{2}{|c|}{ POSTEST } \\
\hline & N. ${ }^{\circ}$ & $\%$ & N. ${ }^{\circ}$ & $\%$ & N. ${ }^{\circ}$ & $\%$ & N. ${ }^{o}$ & $\%$ \\
\hline MALA & 10 & 52.63 & 0 & 0 & 06 & 33.33 & 07 & 38.89 \\
\hline REGULAR & 09 & 47.37 & 02 & 10.53 & 12 & 66.67 & 11 & 61.11 \\
\hline BUENA & 0 & 0 & 17 & 89.47 & 0 & 0 & 0 & 0 \\
\hline TOTAL & 19 & 100.0 & 19 & 100.0 & 18 & 100.0 & 18 & 100.0 \\
\hline
\end{tabular}

Fuente: Prueba de ortografía, elaborada por Guzmán N. Trujillo, 2010.

Tabla 4. Niveles de ortografía alcanzado en el pretest y postest del grupo experimental y control en el uso de la letra "s" de los alumnos del I ciclo de la Escuela de Enfermería de la Universidad Católica Los Ángeles de Chimbote. Sede Trujillo, 2010.

\begin{tabular}{|c|c|c|c|c|c|c|c|c|}
\hline \multirow{3}{*}{$\begin{array}{l}\text { USO DE LA } \\
\text { LETRA "S" }\end{array}$} & \multicolumn{4}{|c|}{ GRUPO EXPERIMENTAL } & \multicolumn{4}{|c|}{ GRUPO CONTROL } \\
\hline & \multicolumn{2}{|c|}{ PRETEST } & \multicolumn{2}{|c|}{ POSTEST } & \multicolumn{2}{|c|}{ PRETEST } & \multicolumn{2}{|c|}{ POSTEST } \\
\hline & N. ${ }^{\circ}$ & $\%$ & N. ${ }^{\circ}$ & $\%$ & N. ${ }^{\circ}$ & $\%$ & N. ${ }^{o}$ & $\%$ \\
\hline MALA & 16 & 84.21 & 0 & 0 & 03 & 16.67 & 03 & 16.67 \\
\hline REGULAR & 03 & 15.79 & 04 & 21.05 & 15 & 83.33 & 15 & 83.33 \\
\hline BUENA & 0 & 0 & 15 & 78.95 & 0 & 0 & 0 & 0 \\
\hline TOTAL & 19 & 100.0 & 19 & 100.0 & 18 & 100.0 & 18 & 100.0 \\
\hline
\end{tabular}

Fuente: Prueba de ortografía, elaborada por Guzmán N. Trujillo, 2010. 
Tabla 5. Niveles de ortografía alcanzado en el pretest y postest del grupo experimental y control en el uso de la letra "c" de los alumnos del I ciclo de la Escuela de Enfermería de la Universidad Católica Los Ángeles de Chimbote, sede Trujillo, 2010.

\begin{tabular}{|c|c|c|c|c|c|c|c|c|}
\hline \multirow{3}{*}{$\begin{array}{l}\text { USO DE LA } \\
\text { LETRA "C" }\end{array}$} & \multicolumn{4}{|c|}{ GRUPO EXPERIMENTAL } & \multicolumn{4}{|c|}{ GRUPO CONTROL } \\
\hline & \multicolumn{2}{|c|}{ PRETEST } & \multicolumn{2}{|c|}{ POSTEST } & \multicolumn{2}{|c|}{ PRETEST } & \multicolumn{2}{|c|}{ POSTEST } \\
\hline & N. ${ }^{\circ}$ & $\%$ & N. ${ }^{o}$ & $\%$ & N. ${ }^{\circ}$ & $\%$ & N. ${ }^{o}$ & $\%$ \\
\hline MALA & 14 & 73.80 & 0 & 0 & 05 & 27.78 & 04 & 22.22 \\
\hline REGULAR & 05 & 26.20 & 03 & 15.79 & 13 & 72.22 & 14 & 77.78 \\
\hline BUENA & 0 & 0 & 16 & 84.21 & 0 & 0 & 0 & 0 \\
\hline TOTAL & 19 & 100.0 & 19 & 100.0 & 18 & 100.0 & 18 & 100.0 \\
\hline
\end{tabular}

Fuente: Prueba de ortografía, elaborada por Guzmán N. Trujillo, 2010.

Tabla 6. Niveles de ortografía alcanzado en el pretest y postest del grupo experimental y control en el uso de la letra "b" y "v" de los alumnos del I ciclo de la Escuela de Enfermería de la Universidad Católica Los Ángeles de Chimbote, sede Trujillo, 2010.

\begin{tabular}{|c|c|c|c|c|c|c|c|c|}
\hline \multirow{3}{*}{$\begin{array}{l}\text { USO DE LA } \\
\text { LETRA "B" y "V" }\end{array}$} & \multicolumn{4}{|c|}{ GRUPO EXPERIMENTAL } & \multicolumn{4}{|c|}{ GRUPO CONTROL } \\
\hline & \multicolumn{2}{|c|}{ PRETEST } & \multicolumn{2}{|c|}{ POSTEST } & \multicolumn{2}{|c|}{ PRETEST } & \multicolumn{2}{|c|}{ POSTEST } \\
\hline & N. ${ }^{o}$ & $\%$ & N. ${ }^{o}$ & $\%$ & N. ${ }^{o}$ & $\%$ & N. ${ }^{\circ}$ & $\%$ \\
\hline MALA & 12 & 63.15 & 0 & 0 & 03 & 16.67 & 04 & 22.22 \\
\hline REGULAR & 07 & 36.84 & 04 & 21.05 & 15 & 83.33 & 14 & 77.78 \\
\hline BUENA & 0 & 0 & 15 & 78.95 & 0 & 0 & 0 & 0 \\
\hline TOTAL & 19 & 100.0 & 19 & 100.0 & 18 & 100.0 & 18 & 100.0 \\
\hline
\end{tabular}

Fuente: Prueba de ortografía, elaborada por Guzmán N. Trujillo, 2010.

Tabla 7. Niveles de ortografía alcanzado en el pretest y postest del grupo experimental y control en el uso de la letra "s" y "c" de los alumnos del I ciclo de la Escuela de Enfermería de la Universidad Católica Los Ángeles de Chimbote, sede Trujillo, 2010.

\begin{tabular}{|c|c|c|c|c|c|c|c|c|}
\hline \multirow{3}{*}{$\begin{array}{c}\text { USO DE LA } \\
\text { LETRA "S" y "C" }\end{array}$} & \multicolumn{4}{|c|}{ GRUPO EXPERIMENTAL } & \multicolumn{4}{|c|}{ GRUPO CONTROL } \\
\hline & \multicolumn{2}{|c|}{ PRETEST } & \multicolumn{2}{|c|}{ POSTEST } & \multicolumn{2}{|c|}{ PRETEST } & \multicolumn{2}{|c|}{ POSTEST } \\
\hline & N. ${ }^{o}$ & $\%$ & N. ${ }^{o}$ & $\%$ & N. ${ }^{\circ}$ & $\%$ & N. ${ }^{\circ}$ & $\%$ \\
\hline MALA & 13 & 68.42 & 0 & 0 & 02 & 11.11 & 03 & 16.67 \\
\hline REGULAR & 06 & 31.58 & 03 & 15.79 & 16 & 88.89 & 15 & 83.33 \\
\hline BUENA & 0 & 0 & 16 & 84.21 & 0 & 0 & 0 & 0 \\
\hline TOTAL & 19 & 100.0 & 19 & 100.0 & 18 & 100.0 & 18 & 100.0 \\
\hline
\end{tabular}

Fuente: Prueba de ortografía, elaborada por Guzmán N. Trujillo, 2010. 
Tabla 8. Niveles de ortografía alcanzado en el pretest y postest del grupo experimental en el uso de la acentuación y tildación de los alumnos del I ciclo de la Escuela de Enfermería de la Universidad Católica Los Ángeles de Chimbote, sede Trujillo, 2010.

\begin{tabular}{|c|c|c|c|c|c|c|c|c|}
\hline \multirow{3}{*}{$\begin{array}{l}\text { ACENTUACIÓN Y } \\
\text { TILDACIÓN }\end{array}$} & \multicolumn{4}{|c|}{ GRUPO EXPERIMENTAL } & \multicolumn{4}{|c|}{ GRUPO CONTROL } \\
\hline & \multicolumn{2}{|c|}{ PRETEST } & \multicolumn{2}{|c|}{ POSTEST } & \multicolumn{2}{|c|}{ PRETEST } & \multicolumn{2}{|c|}{ POSTEST } \\
\hline & N. ${ }^{o}$ & $\%$ & N. ${ }^{o}$ & $\%$ & N. ${ }^{o}$ & $\%$ & N. ${ }^{o}$ & $\%$ \\
\hline MALA & 13 & 68.42 & 0 & 0 & 07 & 38.89 & 07 & 38.89 \\
\hline REGULAR & 06 & 31.58 & 03 & 15.79 & 11 & 61.11 & 11 & 61.11 \\
\hline BUENA & 0 & 0 & 16 & 84.21 & 0 & 0 & 0 & 0 \\
\hline TOTAL & 19 & 100.0 & 19 & 100.0 & 18 & 100.0 & 18 & 100.0 \\
\hline
\end{tabular}

Fuente: Prueba de ortografía, elaborada por Guzmán N. Trujillo, 2010.

Tabla 9. Comparación de promedios para puntajes alcanzados en la ortografía por los alumnos del I ciclo en el grupo control y grupo experimental de la Escuela de Enfermería de la Universidad Católica Los Ángeles de Chimbote, sede Trujillo, 2010.

\begin{tabular}{|c|c|c|c|c|c|c|c|}
\hline \multirow{2}{*}{ Comparación } & & \multicolumn{2}{|c|}{ Promedios } & \multirow{2}{*}{$\begin{array}{c}\text { Valoración } \\
\text { experimental }\end{array}$} & \multirow{2}{*}{$\begin{array}{c}\text { Valor } \\
\text { tabular }\end{array}$} & \multirow{2}{*}{$\begin{array}{l}\text { Decisión } \\
\text { para Ho }\end{array}$} & \multirow{2}{*}{$P: \alpha$} \\
\hline & & 1 & 2 & & & & \\
\hline $\begin{array}{c}\text { Grupo } \\
\text { experimental }\end{array}$ & $\begin{array}{l}\text { Pretest con } \\
\text { post test }\end{array}$ & 3.42 & 7.50 & 7.70 & 1.96 & Se rechaza & $p<0.05$ \\
\hline $\begin{array}{l}\text { Grupo } \\
\text { control }\end{array}$ & $\begin{array}{c}\text { Pre test con } \\
\text { post test }\end{array}$ & 4.83 & 4.58 & 1.18 & 1.96 & Se acepta & $p>0.05$ \\
\hline Pretest & $\begin{array}{c}\text { Grupo } \\
\text { experimental } \\
\text { con grupo } \\
\text { control }\end{array}$ & 3.42 & 4.83 & 3.34 & 1.96 & Se acepta & $P>0.05$ \\
\hline Postest & $\begin{array}{c}\text { Grupo } \\
\text { experimental } \\
\text { con grupo } \\
\text { control }\end{array}$ & 7.50 & 4.58 & 7.61 & 1.96 & Se rechaza & $p<0.05$ \\
\hline
\end{tabular}

Fuente: Prueba de ortografía, elaborada por Guzmán N. Trujillo, 2010.

\section{DISCUSIÓN}

Los resultados muestran que la comparación de promedios para los puntajes alcanzados por los alumnos en el nivel de ortografía, usando la prueba "t" de Student con un nivel de significación del 5\%, confirma la hipótesis de investigación al señalar que, en el grupo experimental, al comparar los promedios obtenidos en el pretest con el promedio obtenido en postest se presentan diferencias significativas entre dichos promedios $(p<0,05)$. No ocurrió lo mismo en el grupo control, pues al no aplicarse el programa "Buena Ortografía sin Esfuerzo" no mejoró la ortografía en estos alumnos del I ciclo de la Escuela de Enfermería de la Universidad Católica Los Ángeles de Chimbote ( $p>0,05)$. 
En el postest se comparó el promedio obtenido por el grupo experimental con el promedio obtenido por el grupo control. Se evidenció una diferencia significativa entre dichos promedios gracias a la intervención del programa "Buena Ortografía sin Esfuerzo" en los alumnos del I ciclo de la Escuela de Enfermería de la Universidad Católica Los Ángeles de Chimbote $(\mathrm{p}<0.05)$.

Estos resultados se asemejan a los encontrados por Vila (2002), en una evaluación de la ortografía en la educación universitaria señalando que la lectura de textos científicos induce a un mejoramiento de la ortografía. Por otro lado, los resultados de la presente investigación indican que los alumnos mejoraron su ortografía literal en cuanto al uso de las letras "V", "B", "C", "S". Es así, que cuando se aplicó el programa "Buena Ortografía sin Esfuerzo", mejoraron su ortografía desde un nivel bajo a un nivel alto en cada una de las letras evaluadas.

Según los resultados encontrados, una de las dificultades más grandes respecto al trabajo con estudiantes con problemas de escritura es aumentar su motivación. Por lo tanto, la enseñanza de escritura debe hacer que los estudiantes escriban sobre algo que les satisfaga. Cuando se enseña a redactar como parte de un ejercicio que solo pone énfasis en la forma correcta de escribir o en pasar un examen, no solo los estudiantes se mantienen sin motivación, sino que tienen menos posibilidades de aprender porque este conocimiento no está relacionado con nada que tenga un propósito significativo (Fraca, 2003).

Lo antes mencionado respalda los resultados en el grupo control, en el que se observó que los alumnos mantuvieron sus niveles de ortografía porque no fueron motivados con ninguna estrategia que permita mejorar su escritura.

Asimismo, es de resaltar que el fracaso del estudiante universitario es uno de las mayores causas de la poca motivación. Los estudiantes que esperan fracasar en tareas de escritura generalmente participan de esta con desgano o simplemente no intervienen (Becerra, 2008).

Lo anterior conduce a afirmar que el alumno aprende porque establece relaciones y encuentra elementos de comprensión y vínculos afectivos con el mundo que le rodea. Por lo tanto, hay que reconocer que esta forma natural de aprendizaje tiene un valor muy importante para el alumno, puesto que lo lleva a relacionarse con el mundo y a satisfacer sus necesidades de una manera placentera, agradable, lúdica y afectiva.

Como se observa, el proceso de la buena ortografía se inicia desde que el alumno nace, pero al llegar al colegio cambian las reglas de juego y, por lo general, el estudiante debe enfrentarse a situaciones y/o actividades que en su mayoría son mecánicas, extensas, planas o con lecturas impuestas (Poggioli, 2006).

La interacción directa con alumnos de educación superior ha demostrado que los aprendizajes no pueden ser ajenos ni aislados de la realidad de los alumnos. Por el contrario, deben satisfacer necesidades, intereses e inquietudes para que tengan un verdadero sentido y significado para él. 
El utilizar técnicas de programación neurolingüística como motivación para mejorar la ortografía, partió de "escuchar" a los alumnos, conocer sus intereses, necesidades, sentimientos e inquietudes; basados en esto, se inició despertando la conciencia del lenguaje a través de ejercicios que les permitan relacionar, hacer asociaciones o establecer semejanzas y diferencias. Los aprendizajes están inmersos en la cotidianidad de los alumnos, esto es lo que hace que tengan sentido y significado para ellos.

Montenegro (2008) señala que los alumnos saben leer y escribir cuando llegan a la universidad, pero lo hacen a su manera; de allí se parte para hacer explícita su intención. Es importante respetar las diferentes etapas de escritura en que está cada alumno. Ellos crean sus propias hipótesis y así van encontrando significado a lo que escriben, comienzan utilizando seudoletras y, poco a poco, las van relacionando con el código alfabético, descubriendo razones válidas de uso.

Por tal razón, es importante resaltar que la motivación a través de la aplicación del Programa "Buena Ortografía sin Esfuerzo" desempeñó un papel decisivo en el proceso de la mejora en el nivel ortográfico.

De manera similar a lo mostrado en los resultados de esta investigación, Rodríguez (2007), llega a resultados afines, indicando que los estudiantes después de aplicado un programa mejoran significativamente su nivel ortográfico.

Es así que el aprendizaje de la escritura en los alumnos se constituyó en un proceso dinámico, en el que la creatividad es muy importante, estimulándose el desarrollo del pensamiento divergente para que los alumnos busquen diferentes alternativas ante una situación dada. Al estimular dicho pensamiento, les damos la oportunidad de crecer siendo seres autónomos, seguros de sí mismos, capaces de tomar decisiones y, de esa forma, los educamos para la vida y no solo para el momento (Pozo, 1999, p. 76).

Todas estas actividades les permiten descubrir el sentido de la lengua escrita y comprender que a través de ella pueden comunicarse, y lo que es aún mejor: expresarse. Queda demostrado que esta forma de trabajo le da un giro a lo tradicional y cambia la posterior relación que el alumno tenga frente a los libros y el lenguaje. Esto posibilita que en un futuro sean mejores lectores y escritores.

El aprendizaje de la escritura es una experiencia que marca la vida del alumno; de ahí la importancia de que pueda acceder a ellas de una forma natural y tranquila. Leer y escribir se convierten en interacciones divertidas y placenteras, en las que el alumno puede disfrutar de sus logros y aprender de sus equivocaciones (Maehr, 1991, p. 3994).

Es así que se comprueba la hipótesis de investigación, la cual señala que el programa "Buena Ortografía sin Esfuerzo" mejora la ortografía en los alumnos del I ciclo de la Escuela de Enfermería de la Universidad Católica Los Ángeles de Chimbote. 


\section{CONCLUSIONES}

1. Después de aplicado el programa "Buena Ortografía sin Esfuerzo" se encontraron mejoras significativas en el uso de las letras "V", "B", "S", "C", así como en la tildación, pasando del nivel regular (31,58\% en el pretest) al nivel bueno (84,21\% en el postest).

2. En el grupo control, en el cual no se aplicó el programa "Buena ortografía sin esfuerzo", no hubo ningún cambio significativo en la aplicación del pre y postest.

3. Se comprueba la hipótesis que sostiene que la aplicación del Programa "Buena ortografía sin esfuerzo" mejora significativamente la ortografía en los alumnos del I ciclo de la Escuela de Enfermería de la Universidad Católica Los Ángeles de Chimbote.

\section{REFERENCIAS BIBLIOGRÁFICAS}

Álvarez, M. (2002). "Vygotski: Hacia la psicología dialéctica". Material utilizado en el Seminario de Psicología Social de la Escuela de Psicología de la Universidad Bolivariana Santiago de Chile.

Becerra, A. (2008). Programa basado en actividades de dibujo libre para estimular el desarrollo de la escritura de la ciudad de Trujillo, Perú. Trujillo: Universidad César Vallejo, Facultad de Educación.

Bravo, L. (2003). Lectura inicial y psicología cognitiva. Santiago: Editorial Universidad Católica.

Calero, M. (1997). Constructivismo: un reto de innovación pedagógica. Lima: San Marcos.

Chloe, M. (2010). La acentuación. Trabajo de ortografía. Slide share.ww. slideshare.net.

Cooper y otros. (2006). Métodos modernos de enseñanza-aprendizaje. México: Editorial Limusa.

De Dios, J (2003). Problemas de aprendizaje. Universidad Pedagógica Nacional. Bogotá: UPN, Facultad de Educación.

Deci, E. L., Vallerand, R. U., Pelletier, L. G., y Ryan, R. M. (1991). Motivation and Education: The Self-Determination Perspective. Educational Psychologist, 26(3/4), pp. 325-346.

Marín, E. y Morales, Ó. Análisis de textos expositivos producidos por estudiantes universitarios desde la perspectiva lingüística discursiva. Educere. Disponible en: https://www.saber.ula.ve/bitstream/123456789/19900/2/articulo6.pdf, pp. 333345. Fecha de consulta: 15 de junio del 2016.

Flavell, J.H. (2000). El desarrollo cognitivo. $2^{\mathrm{a}}$ ed. Madrid: Visor.

Fraca, D. (2003). Dislexia, disortografía y disgrafía. España: Ediciones Pirámide.

Hernández, R., Fernández, C., Baptista, M. (2006). Metodología de la investigación. $4^{\text {a }}$. ed. México: McGraw-Hill. 
Maehr, M. y Midgley, C. (1991). Enhancing Student Motivation: A Schoolwide Approach. Educational Psychologist, 26, pp. 399-427.

Montenegro, S. (2008). Escritura en niños del tercer grado del nivel primario de Distrito de Pelotas, Brasil.

Morales y Fernández. (2005). Estrategias docentes para un aprendizaje significativo. México: McGraw-Hill.

Poggioli, L. (2006). Estrategias de apoyo y motivacionales. Caracas: Fundación Polar.

Portilla, D. (2008). Didáctica aplicada a la educación. Lima: Universidad Nacional Mayor de San Marcos.

Pozo, J. (1999). El aprendizaje estratégico. Madrid: Aula XXI, Santillana.

Rodríguez, G. (2007). Influencia del taller "Aprendiendo a producir cuentos para mejorar el proceso de la escritura en los niños del 2do. grado "B" nivel primario de la Institución Educativa 80229 de la ciudad de Otuzco. Universidad César Vallejo. Facultad de Educación.

Vila, D. (2002). Lectura de fábulas y cuentos en el aprendizaje de la lectoescritura en niños de primer y segundo grado de educación primaria, en el distrito de Carabobo, Venezuela. 